\title{
Adición de aceites vegetales a la dieta de cabras lecheras: efecto sobre la digestibilidad y los resultados productivos
}

\author{
Diet digestibility and production performance in dairy goats consuming plant oils
}

\author{
AL Martínez-Marín*, M Pérez-Hernández, LM Pérez-Alba, D Carrión-Pardo AG Gómez-Castro \\ Departamento de Producción Animal, Universidad de Córdoba, España.
}

\begin{abstract}
SUMMARY
The aim of this study was to investigate the effects of differently unsaturated vegetable oils added to dairy goat diets on apparent digestibility and performance traits. Twelve Malagueña goats were randomly assigned to one of four treatments: no oil (CONTROL), $48 \mathrm{~g} / \mathrm{d}$ of high oleic sunflower oil (GAO), regular sunflower oil (RSFO) or linseed oil (LIN). The basal diet was made of alfalfa hay and a pelleted concentrate, which included the respective oil and chromium as indicator. The experimental period lasted 21 days. The digestibility of dietary components, except for fat, did not differ between treatments $(\mathrm{P}>0.05)$. Oil inclusion in the diet significantly $(\mathrm{P}<0.05)$ increased fat digestibility. There were no significant differences $(\mathrm{P}>0.05)$ in dry matter intake, milk yield and milk composition between treatments. It was concluded that moderate amounts of unsaturated plant oils can be added to dairy goat diets without negative effects on diet digestibility or performance traits.
\end{abstract}

Palabras clave: aceite vegetal, cabras lecheras, digestibilidad, resultados productivos.

Key words: plant oil, dairy goats, digestibility, performance traits.

\section{INTRODUCCIÓN}

El contenido de lípidos de las dietas comunes de los rumiantes (2 a 5\%, de acuerdo con Doreau y Ferlay 1994) puede elevarse para aumentar la concentración energética de la dieta, reducir el riesgo de acidosis ruminal y la caída de la grasa láctea, o modificar los ácidos grasos absorbidos en el intestino delgado y depositados en sus productos (Demeyer y Doreau 1999). El incremento del contenido graso de la dieta requiere la adición de materias primas especialmente ricas en lípidos o fuentes de grasa.

La adición de fuentes de grasa protegidas mediante procedimientos físicos o químicos (encapsulación, hidrogenación, sales de calcio, acilamidas) a la dieta de los rumiantes permite incrementar la cantidad de ácidos grasos dietarios disponibles para la absorción intestinal a la par que se reducen los efectos negativos sobre la población microbiana ruminal (Jenkins y Bridges 2007). Por el contrario, la inclusión de lípidos no protegidos (semillas oleaginosas, aceites y grasas) en la dieta puede afectar negativamente a la población microbiana del rumen (Yang y col 2009) y, por tanto, a la digestión de la dieta y el suministro de sustratos a la glándula mamaria para la síntesis de la leche.

Una revisión de la bibliografía realizada por nuestro grupo (Martínez Marín y col 2011), que incluyó 29 trabajos de investigación y 72 tratamientos experimentales,

Aceptado: 08.09.2011.

* Campus Universitario de Rabanales, Carretera Madrid-Cádiz, km. 396, 14014 Córdoba, España; pa1martm@uco.es concluyó que un aporte igual o inferior a $4 \%$ de grasa extra en la dieta no suele afectar negativamente la digestibilidad total de los carbohidratos fibrosos con independencia del grado de insaturación, forma de presentación y procesado de la fuente de grasa (33 tratamientos sin efecto sobre un total de 40). Cuando la cantidad de grasa extra es mayor de $4 \%$, la ocurrencia de un efecto negativo es más probable (19 tratamientos sobre un total de 32). Ahora bien, la mayoría de los trabajos de investigación que han reportado el efecto del suministro de fuentes de grasa no protegidas a hembras rumiantes lecheras sobre la digestibilidad de la dieta se han realizado con vacas en lactación. El número de investigaciones similares realizadas con cabras en lactación (Maia y col 2006, Silva y col 2007b) es demasiado escaso para concluir que los resultados observados en vacas son similares en las cabras.

La adición de aceites vegetales ricos en AGI a la dieta tiene efectos diferentes sobre la producción y composición de la leche según se trate de vacas, ovejas y cabras (Chilliard y col 2003). Sanz Sampelayo y col (2007) señalaron que la mayor velocidad de tránsito ruminal característica de las ovejas y las cabras podría atenuar el efecto negativo de los AGI sobre la digestión ruminal y la producción de sustratos para la glándula mamaria; o bien, de acuerdo con Chilliard y col (2003), la mayor velocidad de tránsito reduciría la producción ruminal de isómeros del ácido linoleico conjugado que tienen efectos metabólicos directos sobre la síntesis de grasa láctea, como p. ej. 18:2trans-10,cis-12 y 18:2trans-9,cis-11 (Shingfield y Griinari 2007).

El objetivo del presente trabajo fue investigar el efecto de suministrar aceites vegetales de distinto grado de 
insaturación a cabras en lactación sobre la digestibilidad de los componentes de la dieta y la producción y composición de la leche.

\section{MATERIAL Y MÉTODOS}

\section{ALOJAMIENTO, ANIMALES Y MANEJO GENERAL}

Todas las experiencias se llevaron a cabo en el Centro Usuario de Animales de Experimentación CO/5/U ubicado en el edificio del Departamento de Producción Animal de la Universidad de Córdoba. Los animales fueron mantenidos de acuerdo con la normativa española de protección de animales de experimentación (Real Decreto 1201/2005, de 10 de octubre, sobre protección de los animales utilizados para experimentación y otros fines científicos).

Se utilizaron 12 cabras de raza Malagueña (45 \pm 5 días de lactación y 47,2 $\pm 4,2 \mathrm{~kg}$ de peso vivo inicial), alojadas individualmente en jaulas de 1,0 x 1,4 m con suelo permeable a los excrementos y dotadas de comederos y bebederos independientes. El régimen de iluminación fue de 16 horas y la climatización aseguró condiciones termoneutras en el alojamiento. En los meses previos al comienzo de las experiencias todos los animales fueron vacunados y revacunados frente a agalaxia contagiosa, mastitis gangrenosa, enterotoxemia y septicemia hemorrágica (Laboratorios Ovejero, León, España). El ordeño se realizó individualmente, una vez al día, a las 8:30 horas, utilizando el sistema de ordeño mecánico instalado en el alojamiento (DeLaval, Madrid, España). Al finalizar aquel, la leche residual se extrajo mediante ordeño manual. La ración se formuló de acuerdo con las recomendaciones de NRC (2007), se preparó diariamente para cada una de las cabras y se repartió en dos comidas iguales a las 9:30 y 16:00 h.

\section{TRATAMIENTOS Y DIETAS}

Los animales fueron asignados al azar a uno de cuatro tratamientos (cuadro 1): sin aceite añadido a la dieta y adición de $48 \mathrm{~g} / \mathrm{d}$ de aceite de girasol alto oleico, aceite de girasol normal o aceite de lino (dietas CONTROL, GAO, GN y LIN, respectivamente). La dieta base estuvo compuesta por heno de alfalfa y un concentrado granulado en proporción 30/70. La composición del concentrado fue (g/kg): maíz, 375,0; cebada, 374,9, harina de soja, 200,0, premezcla de vitaminas y minerales (Maxi Nutral Ovejas, Nutral, Madrid, España), 30,0; aglomerante (Exal, Tolsa, Madrid, España), 20,0; antioxidante (Luctanox, Lucta, Barcelona, España), 0,1. Los concentrados de los tratamientos con aceite incluyeron $3,85 \%$, sobre peso seco al aire, de uno de los tres aceites. El suministro de concentrado en cada dieta se ajustó de acuerdo con el contenido de aceite para un aporte igual de nutrientes en todos los tratamientos con excepción de la grasa extra. El contenido de energía metabolizable y proteína metabolizable calculado de acuerdo con NRC (2007) fue 2,67 Mcal/kg y $123 \mathrm{~g} / \mathrm{kg}$ en la dieta CONTROL y 2,77 Mcal/kg y $119 \mathrm{~g} / \mathrm{kg}$ en las dietas GAO, GN y LIN. El período experimental duró 21 días, incluyendo la adaptación a los tratamientos y la toma de muestras.

\section{MEDIDAS, TOMA DE MUESTRAS Y ANÁLISIS}

El peso (PV) de las cabras se controló al comienzo y al final del período experimental mediante una báscula MOBBA 6010 (MOINCASA, Barcelona, España) con una precisión de $\pm 0,1 \mathrm{~kg}$. Las pesadas se realizaron siempre inmediatamente después del ordeño y antes de la primera comida del día.

El consumo de alimentos se registró diariamente para cada animal y se calculó como la diferencia entre la ración ofrecida y los residuos recogidos en el comedero al día siguiente, antes de la comida de la mañana. Los ingredientes de la dieta fueron muestreados al comienzo del experimento y almacenados junto a los residuos a $-20{ }^{\circ} \mathrm{C}$ hasta el análisis.

La digestibilidad se determinó utilizando cromo como indicador externo (Udén y col 1980). El cromo fue ligado a harina de trigo mezclando homogéneamente ésta con dicromato potásico diluido en agua y calentando la masa húmeda obtenida, protegida del contacto con el aire, durante 24 h en estufa a $100{ }^{\circ} \mathrm{C}$. El producto así preparado se añadió al concentrado en una cantidad de $2 \%$. La recolección de heces de recto se realizó durante cuatro días a intervalos de cuatro horas, atrasando la recogida una hora cada día. Muestras de $25 \mathrm{~g}$ de heces se desecaron en estufa a $103^{\circ} \mathrm{C}$, posteriormente se mezclaron alícuotas de las muestras de cada cabra para obtener una muestra compuesta para el análisis. El contenido de cromo del concentrado y las heces se determinó por colorimetría según el procedimiento descrito por Fenton y Fenton (1979). La digestibilidad se calculó según la fórmula: Digestibilidad $=100-[100 *$ (\%indicador en el alimento / \%indicador en las heces) * (\%nutriente en las heces / \%nutriente en el alimento)].

Los procedimientos descritos por AOAC (2006) fueron utilizados para determinar la materia seca (MS) y la fibra bruta en los alimentos, los residuos y las heces, y las cenizas, la proteína bruta $(\mathrm{PB})$ y la grasa por hidrólisis ácida previa (GHA) en los alimentos y las heces. La fibra neutrodetergente (FND) fue analizada por el método de Van Soest y col (1991). El almidón se determinó por polarimetría según ISO (2000ª). Los carbohidratos no fibrosos (CNF) fueron calculados por diferencia (Mertens 1997). Los ácidos grasos de los aceites fueron metilados de acuerdo con ISO $\left(2000^{\mathrm{b}}\right)$ y cuantificados por cromatografía gaseosa mediante el procedimiento descrito por ISO (1990).

El último día del período experimental se recogieron dos muestras de leche de cada animal. Una muestra se utilizó para la determinación de la composición química, mediante análisis en un Milko-Scan FT120 (Foss Electric, Hillerød, Denmark) calibrado para leche de cabra. La 
Cuadro 1. Dietas experimentales.

Experimental diets.

\begin{tabular}{|c|c|c|c|c|}
\hline \multirow{2}{*}{ Item } & \multicolumn{4}{|c|}{ Tratamientos $^{1}$} \\
\hline & CONTROL $^{2}$ & GAO & GN & LIN \\
\hline \multicolumn{5}{|l|}{ Dieta, $\mathrm{g} / \mathrm{d}$} \\
\hline Heno de alfalfa & 600 & 600 & 600 & 600 \\
\hline Concentrado $^{3}$ & 1.200 & 1.200 & 1.200 & 1.200 \\
\hline Aceite de girasol alto oleico ${ }^{4}$ & - & 48 & - & - \\
\hline Aceite de girasol normal ${ }^{4}$ & - & - & 48 & - \\
\hline Aceite de lino $0^{4}$ & - & - & - & 48 \\
\hline \multicolumn{5}{|l|}{ Composición química } \\
\hline Materia seca, $\%$ & 90,6 & 90,5 & 91,0 & 91,2 \\
\hline Proteína bruta, \% MS & 17,0 & 16,4 & 16,4 & 16,5 \\
\hline Fibra bruta, \% MS & 15,1 & 14,9 & 14,6 & 14,7 \\
\hline Cenizas, \% MS & 7,6 & 7,5 & 7,5 & 7,5 \\
\hline Fibra neutrodetergente, $\% \mathrm{MS}$ & 28,2 & 27,5 & 27,0 & 26,9 \\
\hline Carbohidratos no fibrosos, $\%$ & 44,8 & 43,0 & 43,6 & 43,3 \\
\hline Almidón, \% MS & 34,5 & 33,9 & 33,5 & 33,3 \\
\hline Grasa hidrólisis ácida, \% MS & 2,4 & 5,6 & 5,5 & 5,8 \\
\hline $\mathrm{Cromo}^{5}, \% \mathrm{MS}$ & 0,17 & 0,15 & 0,15 & 0,15 \\
\hline \multicolumn{5}{|c|}{ Ácidos grasos aportados por el aceite, g/d } \\
\hline $16: 0$ & - & 1,8 & 2,9 & 2,6 \\
\hline 18:0 & - & 1,4 & 2,0 & 1,8 \\
\hline 18:1cis-9 & - & 41,0 & 14,2 & 10,0 \\
\hline $18: 2 n-6$ & - & 2,7 & 27,9 & 8,0 \\
\hline $18: 3 n-3$ & - & - & - & 23,9 \\
\hline
\end{tabular}

GAO, GN y LIN: dietas enriquecidas con aceite de girasol alto oleico, girasol normal y lino, respectivamente.

2 La dieta CONTROL aportó 5,1, 0,8, 5,5, 14,4 y 3,4 g/d de 16:0, 18:0, 18:1cis-9, 18:2n-6 y 18:3n-3, respectivamente, calculado de acuerdo con INRA (2002).

3 Ver texto.

4 Incluido en el concentrado respectivo.

5 Expresado como óxido de cromo III.

1 GAO, GN and LIN: diets enriched with high oleic sunflower oil, regular sunflower oil and linseed oil, respectively.

2 CONTROL supplied 5.1, 0.8, 5.5, 14.4 and $3.4 \mathrm{~g} / \mathrm{d}$ of 16:0, 18:0, 18:1 cis-9, 18:2n-6 and 18:3n-3, respectively, calculated according to INRA (2002).

3 See text.

4 Included in the respective concentrate.

5 Expressed as chromium oxide III.

muestra restante se conservó congelada a $-20{ }^{\circ} \mathrm{C}$. La producción individual de leche se midió en cada uno de los tres últimos días del período experimental y se expresó en una base de $24 \mathrm{~h}$.

\section{ANÁLISIS ESTADÍSTICO}

El estudio estadístico se realizó con el procedimiento GLM de SAS 9.1 (SAS Inst Inc, Cary, NC). El modelo utilizado para el análisis de los datos de digestibilidad fue: $Y i j=\mu+\alpha i+\varepsilon i j$, donde $Y i j=$ variable dependiente; $\mu=$ media general; $\alpha i=$ efecto del tratamiento ( $i=1$ a 4 ); $\varepsilon i j=$ error residual. El modelo utilizado para el análisis de los datos productivos fue: $Y i j=\mu+\alpha i+\beta X i j+\varepsilon i j$, donde Yij = variable dependiente; $\mu=$ media general; $\alpha \mathrm{i}=$ efecto del tratamiento (i = 1 a 4); $\beta \mathrm{Xij}=$ covariable (valor preexperimental de la variable dependiente); $\varepsilon \mathrm{ij}=$ error residual. La comparación de las medias se realizó por el test de Tukey. El nivel de significación se estableció en $\mathrm{P}<0,05$ en todas las pruebas. Los resultados presentados en los cuadros están expresados como medias de mínimos cuadrados, ajustados por la covariable en el caso de los datos productivos.

\section{RESULTADOS}

El consumo de aceite fue inferior al previsto $(48 \mathrm{~g} / \mathrm{d})$ pero no hubo diferencias significativas $(P>0,05)$ entre tratamientos: 42,2, 44,2 y 40,9 g/d (EE = 4,5), en los tratamientos GAO, GN y LIN respectivamente. Por otro lado, el consumo de AGI fue claramente mayor en las dietas con aceite añadido y hubo diferencias apreciables entre estas en el grado de insaturación de la grasa aportada. El ácido oleico representó casi el 50\% de los ácidos grasos totales aportados por la dieta GAO. El ácido linoleico supuso en torno a $40 \%$ de los ácidos grasos totales aportados por la 
dieta GN, duplicando el contenido de ácido oleico. En la dieta LIN, el aporte de ácido $\alpha$-linolénico fue ligeramente mayor que el del ácido linoleico y, a su vez, el aporte de ácido oleico fue menos de la mitad del de este (cuadro 1).

Los resultados de consumo, excreción fecal y digestión de los componentes de las dietas y coeficientes de digestibilidad total aparente de estos se muestran en el cuadro 2 .
No hubo diferencias significativas $(\mathrm{P}>0,05)$ por efecto del tratamiento en el consumo de los componentes de la dieta ni en los parámetros digestivos calculados a partir del coeficiente de digestibilidad total aparente, con excepción de la GHA. La cantidad consumida y digerida y el coeficiente de digestibilidad total aparente de la GHA fueron significativamente mayores $(\mathrm{P}<0,05)$ en las dietas con

Cuadro 2. Digestión de los componentes de dietas para cabras en lactación sin aceite añadido (CONTROL) o con 48 g/d de aceite de girasol alto oleico (GAO), aceite de girasol normal (GN) o aceite de lino (LIN). oil (GN) or linseed oil (LIN)

Nutrient digestion of dairy goat diets which included none (CONTROL), or $48 \mathrm{~g} / \mathrm{d}$ of high oleic sunflower oil (GAO), regular sunflower

\begin{tabular}{|c|c|c|c|c|c|}
\hline \multirow{2}{*}{ Item $^{\dagger}$} & \multicolumn{4}{|c|}{ Tratamientos } & \multirow{2}{*}{$\mathrm{EE}$} \\
\hline & CONTROL & GAO & GN & LIN & \\
\hline \multicolumn{6}{|l|}{ Materia orgánica } \\
\hline Ingerido, g/d & 1.441 & 1.437 & 1.467 & 1.396 & 28 \\
\hline Excretado, g/d & 359 & 293 & 368 & 311 & 22 \\
\hline Digerido, $\mathrm{g} / \mathrm{d}$ & 1.083 & 1.143 & 1.100 & 1.085 & 25 \\
\hline Digestibilidad, \% & 75,6 & 79,1 & 75,7 & 77,2 & 1,3 \\
\hline \multicolumn{6}{|l|}{ Proteína bruta } \\
\hline Ingerido, g/d & 265 & 255 & 259 & 251 & 5 \\
\hline Excretado, g/d & 64 & 57 & 69 & 64 & 4 \\
\hline Digerido, $\mathrm{g} / \mathrm{d}$ & 201 & 198 & 191 & 187 & 5 \\
\hline Digestibilidad, \% & 75,7 & 78,1 & 73,4 & 74,5 & 1,2 \\
\hline \multicolumn{6}{|l|}{ Fibra bruta } \\
\hline Ingerido, g/d & 235 & 232 & 231 & 223 & 4 \\
\hline Excretado, g/d & 126 & 129 & 126 & 116 & 9 \\
\hline Digerido, $\mathrm{g} / \mathrm{d}$ & 109 & 103 & 105 & 107 & 8 \\
\hline Digestibilidad, \% & 44,8 & 47,9 & 42,7 & 49,5 & 3,5 \\
\hline \multicolumn{6}{|l|}{ Fibra neutrodetergente } \\
\hline Ingerido, $\mathrm{g} / \mathrm{d}$ & 439 & 429 & 426 & 410 & 9 \\
\hline Excretado, g/d & 207 & 230 & 218 & 200 & 13 \\
\hline Digerido, $\mathrm{g} / \mathrm{d}$ & 233 & 198 & 209 & 210 & 11 \\
\hline Digestibilidad, \% & 51,3 & 49,5 & 46,1 & 52,9 & 2,4 \\
\hline \multicolumn{6}{|c|}{ Carbohidratos no fibrosos } \\
\hline Ingerido, $\mathrm{g} / \mathrm{d}$ & 698 & 670 & 689 & 659 & 15 \\
\hline Excretado, g/d & 46 & 0,0 & 38 & 29 & 8 \\
\hline Digerido, $\mathrm{g} / \mathrm{d}$ & 652 & 670 & 651 & 630 & 15 \\
\hline Digestibilidad, $\%$ & 94,3 & 99,7 & 96,0 & 94,7 & 1,2 \\
\hline \multicolumn{6}{|c|}{ Grasa por hidrólisis ácida } \\
\hline Ingerido, $\mathrm{g} / \mathrm{d}$ & $39^{\mathrm{b}}$ & $81^{\mathrm{a}}$ & $92^{\mathrm{a}}$ & $75^{\mathrm{a}}$ & 6 \\
\hline Excretado, g/d & 17 & 13 & 21 & 17 & 1 \\
\hline Digerido, $\mathrm{g} / \mathrm{d}$ & 22 & 69 & 72 & 59 & 6 \\
\hline Digestibilidad, \% & $58,5^{\mathrm{b}}$ & $81,9^{\mathrm{a}}$ & $79,4^{\mathrm{a}}$ & $76,2^{\mathrm{a}}$ & 3,4 \\
\hline $\mathrm{NDT}^{1}, \%$ & 72,8 & 79,6 & 76,2 & 77,0 & 1,3 \\
\hline $\mathrm{EM}^{2}, \mathrm{kcal} / \mathrm{kg}$ & 2,62 & 2,87 & 2,74 & 2,77 & 0,09 \\
\hline
\end{tabular}

Todos los valores están expresados sobre materia seca.

Nutrientes digestibles totales.

Energía metabolizable; ambos calculados de acuerdo con NRC (2007).

All values are expressed in dry matter basis.

Total digestible nutrients.

Metabolizable energy; both calculated according to NRC (2007). 
aceite añadido. El almidón fue completamente digerido, hallándose la cantidad en heces por debajo del límite de capacidad de detección del método analítico $(<5 \%$ MS). Los contenidos de nutrientes digestibles totales (NDT) y energía metabolizable (EM) de las dietas, calculados a partir de los datos de digestibilidad, no fueron significativamente distintos $(\mathrm{P}>0,05)$ entre los tratamientos.

Los resultados productivos se muestran en el cuadro 3. Los tratamientos no afectaron significativamente $(\mathrm{P}>0,05)$ al consumo de materia seca (CMS) total, al consumo de concentrado, a la producción lechera ni a la composición de la leche. El consumo de heno de alfalfa fue significativamente mayor en los tratamientos CONTROL y GN, menor en el tratamiento LIN e intermedio en el tratamiento GAO. Aunque el contenido de grasa láctea no mostró diferencias significativas entre los tratamientos $(\mathrm{P}>0,05)$, se observaron diferencias numéricas paralelas a las diferencias observadas en el consumo de heno de alfalfa. El porcentaje graso fue numéricamente mayor en los tratamientos CONTROL y GN, menor en el tratamiento LIN e intermedio en el tratamiento GAO.

\section{DISCUSIÓN}

\section{DIGESTIBILIDAD}

La ausencia de efectos negativos de la adición de los aceites a la dieta control sobre la digestión de la PB (cuadro 2) es un hallazgo común en los experimentos de digestibilidad. De forma general, la inclusión de fuentes de grasa no protegidas en la dieta de los rumiantes no afecta (Hristov y col 2005) o aumenta la digestibilidad aparente de la PB (Beauchemin y col 2007). Doreau y Ferlay (1995) indicaron que los lípidos no protegidos no afectan al flujo duodenal de nitrógeno no amoniacal ya sea de origen microbiano o dietético. Según estos autores, este hecho indicaría que el efecto de los lípidos sobre la degradación de la $\mathrm{PB}$ en el rumen y la síntesis microbiana es mínimo. Sin embargo, Silva y col $\left(2007^{\mathrm{b}}\right)$ observaron que la adición de 4,5\% de grasa extra en forma de aceite o semilla de soja a la dieta de cabras en lactación redujo la digestibilidad aparente de la PB. Estos autores atribuyeron el efecto observado a una menor degradación de la proteína dietética, por reducción de los protozoos ruminales, y, por tanto, una mayor contribución de la misma al nitrógeno fecal. Lo contrario sería más probable de acuerdo con Jouany (1996) quien observó que la reducción de la población protozoaria del rumen a menudo resulta en un aumento del número de bacterias y mayor entrada de proteína microbiana al intestino delgado. Este hecho podría resultar también en una reducción de la digestibilidad aparente de la PB, pero la causa sería una mayor excreción fecal de nitrógeno microbiano no digerido.

La adición de fuentes de grasa no protegidas a la dieta de los rumiantes, especialmente aceites vegetales, se relaciona comúnmente con una reducción de la digestibilidad de las paredes vegetales (Doreau y Chilliard 1997). La causa estriba en el efecto negativo, bien conocido, de los aceites sobre los protozoos y las bacterias fibrolíticas (Yang y col 2009). No obstante, la ausencia de efectos negativos de los aceites sobre la digestibilidad de la FND observada en el presente trabajo (cuadro 2) está de acuerdo con experimentos publicados en los que la adición de aceites con similar grado de insaturación (colza, soja y lino) a la dieta de ovejas y vacas fue inferior a $4 \%$ MS (Zervas y col 1998, Ueda y col 2003). Cuando la cantidad de grasa extra añadida a la dieta es superior a $4 \%$ MS, la ocurrencia de efectos negativos sobre la digestibilidad de la FND es más frecuente en todas las especies rumiantes con independencia del grado de insaturación (Hess y col 2001, Maia y col 2006, Martin y col 2008). Por otro lado, una menor digestión ruminal de la FND compensada totalmente por

Cuadro 3. Resultados productivos de cabras en lactación cuya dieta no incluyó aceite (CONTROL) o aportó 48 g/d de aceite de girasol alto oleico (GAO), aceite de girasol normal (GN) o aceite de lino (LIN).

Performance traits of dairy goats fed diets which included none (CONTROL) or $48 \mathrm{~g} / \mathrm{d}$ of high oleic sunflower oil (GAO), regular sunflower oil (GN) or linseed oil (LIN).

\begin{tabular}{|c|c|c|c|c|c|}
\hline \multirow{2}{*}{ Item } & \multicolumn{4}{|c|}{ Tratamientos } & \multirow{2}{*}{$\mathrm{EE}$} \\
\hline & CONTROL & GAO & GN & LIN & \\
\hline Peso vivo, $\mathrm{kg}$ & 44,6 & 45,7 & 49,5 & 46,7 & 1,0 \\
\hline \multicolumn{6}{|l|}{ Consumo de materia seca } \\
\hline $\begin{array}{l}\text { Total, g/d } \\
\text { Concentrado, g/d } \\
\text { Heno de alfalfa, g/d }\end{array}$ & $\begin{array}{r}1.560 \\
1.000 \\
559^{\mathrm{a}}\end{array}$ & $\begin{array}{l}1.553 \\
1.018 \\
535^{\mathrm{ab}}\end{array}$ & $\begin{array}{l}1.586 \\
1.015 \\
571^{\mathrm{a}}\end{array}$ & $\begin{array}{l}1.510 \\
1.028 \\
481^{\mathrm{b}}\end{array}$ & $\begin{array}{l}30 \\
30 \\
10\end{array}$ \\
\hline \multicolumn{6}{|l|}{ Leche } \\
\hline $\begin{array}{l}\text { Producción, g/d } \\
\text { Grasa, \% } \\
\text { Proteína, \% } \\
\text { Lactosa, \% }\end{array}$ & $\begin{array}{r}1.719 \\
5,52 \\
2,66 \\
4,34\end{array}$ & $\begin{array}{r}1.879 \\
4,99 \\
2,76 \\
4,75\end{array}$ & $\begin{array}{r}2.074 \\
5,64 \\
2,99 \\
4,45\end{array}$ & $\begin{array}{r}1.793 \\
4,71 \\
2,55 \\
4,36\end{array}$ & $\begin{array}{r}112 \\
0,27 \\
0,11 \\
0,05\end{array}$ \\
\hline
\end{tabular}


la digestión posruminal no puede ser descartada en alguno de los tratamientos con aceite de nuestro trabajo a pesar del bajo nivel de grasa extra en la dieta (Murphy y col 1987).

La ausencia de efectos negativos sobre la digestibilidad de los CNF en los tratamientos con aceite (cuadro 2) coincide con los resultados obtenidos por Maia y col (2006) y Silva y col $\left(2007^{\mathrm{ab}}\right)$ con cabras, Martin y col (2008) con bovinos y Zervas y col (1998) con ovinos. Los valores obtenidos en todos los tratamientos del presente trabajo fueron muy altos y superiores a los observados por Maia y col (2006) y Silva y col $\left(2007^{\mathrm{a}, \mathrm{b}}\right)$, probablemente debido a la elevada proporción de almidón en los CNF de nuestras dietas. Además, la digestibilidad total aparente de los CNF en los rumiantes es una función lineal de su contenido en la dieta (Detmann y col 2008).

El aumento de la digestibilidad aparente de la grasa observado en los tratamientos con aceite comparados con el control (cuadro 2) está de acuerdo con Palmquist (1991). Este autor señaló que el aumento del consumo de grasa incrementa la digestibilidad aparente de los lípidos debido a la dilución de la grasa endógena fecal y los lípidos no saponificables de los alimentos pero ocasiona una disminución de la digestibilidad verdadera. En este sentido, debe señalarse que la digestibilidad intestinal de la grasa disminuye linealmente al aumentar su consumo debido principalmente a la baja digestibilidad del ácido esteárico (Plascencia y col 2003), cuyo flujo duodenal se incrementa marcadamente en las dietas ricas en AGI por efecto de la biohidrogenación ruminal de estos (Sauvant y Bas 2001).

\section{RESULTADOS PRODUCTIVOS}

La ausencia de efecto negativo de los tratamientos sobre el CMS (cuadro 3) concuerda con los trabajos de Mir y col (1999) con cabras y Ollier y col (2009) con vacas. El efecto negativo de las fuentes de grasa sobre el CMS es más común en vacas (Chilliard y col 2001). De acuerdo con Chilliard y col (1993), la inclusión de fuentes de grasa en la dieta podría reducir el CMS por enlentecimiento del vaciado ruminal, como consecuencia de un efecto negativo sobre la digestión ruminal de las paredes vegetales, o a través de un efecto metabólico de los ácidos grasos de cadena larga aportados. La relación entre la disminución de la digestión ruminal de la FND y el CMS en cabras no ha sido estudiada. En los trabajos en que se midió el efecto de las fuentes de grasa sobre la digestibilidad total de la FND y el CMS, la relación entre ambos parámetros fue contradictoria. Maia y col (2006) no observaron diferencias en el CMS pero la digestibilidad total de la FND fue de media $24 \%$ inferior en los tratamientos con aceite añadido respecto al control. Silva y col $\left(2007^{\mathrm{b}}\right)$ observaron que el aceite de soja redujo un $10 \%$ la digestibilidad total de la FND y 0,3 kg/d el CMS respecto a la dieta control; sin embargo, la semilla de soja molida no afectó a la digestibilidad de la FND pero redujo el CMS en la misma cantidad que el aceite. Por otro lado, los trabajos de Teh y col (1994) y Brown-Crowder y col (2001), en los que se incluyeron niveles crecientes de grasa extra en la dieta (hasta $9 \%$ de jabón cálcico de palma y $6 \%$ de sebo, respectivamente), sugieren que, si existe un efecto metabólico de la grasa sobre el CMS, el límite superior es más elevado que en vacas. En dichos trabajos, el consumo de grasa extra en relación al PV fue muy superior (3,6 y 2,4 g/kg, respectivamente) al que ocasiona reducción significativa del CMS en vacas (1,5 g/kg; Drackley y col 2007).

Cuando se añaden fuentes de grasa a la dieta de cabras en mitad de la lactación, el único cambio observado usualmente es un incremento del contenido y la producción de grasa láctea (Chilliard y col 2003). En el presente trabajo ni la producción de leche ni su composición fueron afectadas por los tratamientos (cuadro 3). La ausencia de diferencias en la producción lechera entre el tratamiento CONTROL y los tratamientos con aceite, a pesar de que el CMS fue similar y el aporte de EM fue 3,7\% mayor en los segundos, podría explicarse, al menos en parte, por diferencias en la digestibilidad intestinal de la grasa consumida como se ha mencionado más arriba. Plascencia y col (2003) observaron en terneros que el valor de energía neta asignado comúnmente a la grasa es válido cuando la grasa consumida total es inferior a $1 \mathrm{~g} / \mathrm{kg} \mathrm{PV}$. En el presente trabajo, el consumo total de grasa fue $0,85,1,78,1,95$ y $1,61 \mathrm{~g} / \mathrm{kg}$ PV $(\mathrm{EE}=0,13)$ y la digestibilidad intestinal de aquella, aplicando la ecuación de Plascencia y col (2003), fue 80,1, 72,4, 71,6 y 73,6\% en los tratamientos CONTROL, GAO, GN y LIN, respectivamente. El hecho de que el contenido de grasa láctea fuera paralelo al menor consumo de heno de alfalfa en los tratamientos GAO y, sobre todo, LIN está de acuerdo con Lu y col (2008) quienes indicaron que existe una relación clara entre la reducción del consumo de forraje, medido como consumo de fibra acidodetergente, y la disminución de contenido de grasa láctea en cabras, que estaría mediada por la disminución del tiempo de masticación, el pH ruminal y la ratio acetato/propionato. Los trabajos de Li y col (2007) y Cheng y col (2009) con cabras han establecido que una ratio acetato/propionato adecuada favorece la síntesis de grasa láctea.

Se concluye que en las condiciones del presente trabajo, la inclusión de niveles moderados de aceite en la dieta de cabras en lactación no tuvo efectos negativos sobre la digestibilidad de los nutrientes ni los parámetros productivos, con independencia del grado de insaturación. Este hecho es ventajoso cuando el objetivo de suministrar aceite a los animales sea modificar el perfil de ácidos grasos de la leche. 


\section{RESUMEN}

El objetivo del presente trabajo fue investigar el efecto de la adición de aceite vegetal y su grado de insaturación sobre la digestibilidad aparente y los parámetros productivos de cabras en lactación. Doce cabras de raza Malagueña fueron asignadas al azar a uno de cuatro tratamientos: sin aceite (CONTROL) y $48 \mathrm{~g} / \mathrm{d}$ de aceite de girasol alto oleico (GAO), aceite de girasol normal (GN) o aceite de lino (LIN). La dieta base estuvo compuesta por heno de alfalfa y un concentrado en el que se incluyó el aceite correspondiente y cromo como indicador. El período experimental duró 21 días. La digestibilidad de los componentes de la dieta, con excepción de la grasa, no fue diferente entre los tratamientos $(\mathrm{P}>0,05)$. La inclusión de aceite en la dieta aumentó significativamente la digestibilidad de la grasa $(\mathrm{P}<0,05)$. No hubo diferencias significativas $(\mathrm{P}>0,05)$ en el consumo de alimentos y la producción y composición de la leche entre los tratamientos. Se concluyó que es posible incluir cantidades moderadas de aceite vegetal rico en ácidos grasos insaturados en la dieta de cabras en lactación sin ocasionar efectos negativos sobre la digestibilidad de los nutrientes o los parámetros productivos.

\section{REFERENCIAS}

AOAC, Association of Official Agricultural Chemists. 2006. Official Methods of Analysis of the Association of Official Agricultural Chemists. $18^{\text {th }}$ ed. 1st rev. AOAC Int, Gaithersburg, USA.

Beauchemin KA, SM McGinn, HV Petit. 2007. Methane abatement strategies for cattle: Lipid supplementation of diets. Can J Anim Sci 87, 431-440.

Brown-Crowder IE, SP Hart, M Cameron, T Sahlu, AL Goetsch. 2001. Effects of dietary tallow level on performance of Alpine does in early lactation. Small Rumin Res 39, 233-241.

Cheng GM, XY Lin, FC Li, FM Chen, ZH Wang, JS Liu, GH Fu. 2009. The influence of acetate and propionate molar ratio in ruminally infused volatile fatty acid mixtures on milk fat synthesis in lactating goats. Acta Vet Zootech Sinica 40, 1028-1036.

Chilliard Y, M Doreau, G Gagliostro, Y Elmeddah. 1993. Addition de lipides protégés (encapsulés ou savons de calcium) à la ration de vaches laitières. Effets sur les performances et la composition du lait. INRA Prod Anim 6, 139-150.

Chilliard Y, A Ferlay, M Doreau. 2001. Effect of different types of forages, animal fat or marine oils in cow's diet on milk fat secretion and composition, especially conjugated linoleic acid (CLA) and polyunsaturated fatty acids. Livest Prod Sci 70, 31-48.

Chilliard Y, A Ferlay, J Rouel, G Lamberet. 2003. A review of nutritional and physiological factors affecting goat milk lipid synthesis and lipolyisis. J Dairy Sci 86, 1751-1770.

Demeyer D, M Doreau. 1999. Targets and procedures for altering ruminant meat and milk lipids. Proc Nutr Soc 58, 593-607.

Detmann E, SC Valadares Filho, DS Pina, LT Henriques, MF Paulino, KA Magalhães, PA Silva, ML Chizzotti. 2008. Prediction of the energy value of cattle diets based on the chemical composition of the feeds under tropical conditions. Anim Feed Sci Technol 143, 127-147.

Doreau M, A Ferlay. 1994. Digestion and utilisation of fatty acids by ruminants. Anim Feed Sci Technol 45, 379-396.

Doreau M, A Ferlay. 1995. Effect of dietary lipids on nitrogen metabolism in the rumen: a review. Livest Prod Sci 43, 97-110.

Doreau M, Y Chilliard. 1997. Digestion and metabolism of dietary fat in farm animals. Br J Nutr 78, S15-S35.

Drackley JK, TR Overton, G Ortiz-Gonzalez, AD Beaulieu, DM Barbano, JM Lynch, EG Perkins. 2007. Responses to increasing amounts of high-oleic sunflower fatty acids infused into the abomasum of lactating dairy cows. J Dairy Sci 90, 5165-5175.

Fenton TW, M Fenton. 1979. An improved procedure for the determination of chromic oxide in feed and feces. Can J Anim Sci 59, 631-634.
Hess BW, MB Whitney, DC Rule. 2001. Site and extent of digestion by beef heifers fed medium-quality hay and supplemental corn or soybean oil. Proc West Sect Am Soc Anim Sci 52, 469-472.

Hristov AN, LR Kennington, MA McGuire, CW Hunt. 2005. Effect of diets containing linoleic acid- or oleic acid-rich oils on ruminal fermentation and nutrient digestibility, and performance and fatty acid composition of adipose and muscle tissues of finishing cattle. J Anim Sci 83, 1312-1321.

INRA, Institut National de la Recherche Agronomique. 2002. Tables de composition et de valeur nutritive des matières premières destinées aux animaux d'élevage. INRA Éditions, Paris, France.

ISO, International Organization for Standardization. 1990. Animal and vegetable fats and oils - Analysis by gas chromatography of methyl esters of fatty acids. International Organization for Standardization, Geneva, Switzerland.

ISO, International Organization for Standardization. 2000 ${ }^{\mathrm{a}}$. Animal feeding stuffs - Determination of starch content - Polarimetric method. International Organization for Standardization, Geneva, Switzerland.

ISO, International Organization for Standardization. $2000^{\mathrm{b}}$. Animal and vegetable fats and oils - Preparation of methyl esters of fatty acids. International Organization for Standardization, Geneva, Switzerland.

Jenkins TC, WC Bridges Jr. 2007. Protection of fatty acids against ruminal biohydrogenation in cattle. Eur J Lipid Sci Technol 109, 778-789.

Jouany JP. 1996. Effect of rumen protozoa on nitrogen utilization by ruminants. J Nutr 126, 1335S-1346S.

Li B, ZH Wang, FC Li, XY Lin. 2007. Milk fat content was changed by ruminal infusion of mixed VFAs solutions with different acetate/ propionate ratios in lactating goats. Small Rumin Res 72, 11-17.

Lu CD, JR Kawas, OG Mahgoub. 2008. Recent advancements in fiber digestion and utilization in goats. Tropical and Subtropical Agroecosystems 9, 65-72.

Maia FJ, AF Branco, GF Mouro, SM Coneglian, GT Santos, TF Minella, KC Guimarães. 2006. Inclusão de fontes de óleo na dieta de cabras em lactação: digestibilidade dos nutrientes e parãmetros ruminais e sangüíneos. Rev Bras Zootec 35, 1496-1503.

Martin C, J Rouel, JP Jouany, M Doreau, Y Chilliard. 2008. Methane output and diet digestibility in response to feeding dairy cows crude linseed, extruded linseed, or linseed oil. J Anim Sci 86, 2642-2650.

Martínez Marín AL, M Pérez Hernández, LM Pérez Alba, G Gómez Castro, D Carrión Pardo. 2011. Efecto de las fuentes de grasa sobre la digestión de la fibra en los rumiantes. REDVET Rev electrón vet $12,1-22$.

Mertens DR. 1997. Creating a system for meeting the fiber requirements of dairy cows. J Dairy Sci 80, 1463-1481.

Mir Z, LA Goonewardene, E Okine, S Jaegar, HD Scheer. 1999. Effect of feeding canola oil on constituents, conjugated linoleic acid (CLA) and long chain fatty acids in goats milk. Small Rumin Res 33, 137-143.

Murphy M, P Udén, DL Palmquist, H Wiktorsson. 1987. Rumen and total diet digestibilities in lactating cows fed diets containing full-fat rapeseed. J Dairy Sci 70, 1572-1582.

NRC, National Research Council. 2007. Nutrient requirements of small ruminants. National Academy Press, Washington DC, USA.

Ollier S, C Leroux, A de la Foye, L Bernard, J Rouel, Y Chilliard. 2009. Whole intact rapeseeds or sunflower oil in high-forage or highconcentrate diets affects milk yield, milk composition, and mammary gene expression profile in goats. J Dairy Sci 92, 5544-5560.

Palmquist DL. 1991. Influence of source and amount of dietary fat on digestibility in lactating cows. J Dairy Sci 74, 1354-1364.

Plascencia A, GD Mendoza, C Vásquez, RA Zinn. 2003. Relationship between body weight and level of fat supplementation on fatty acid digestion in feedlot cattle. J Anim Sci 81, 2653-2659.

Sanz Sampelayo MR, I Chilliard, P Schmidely, J Boza. 2007. Influence of type of diet on the fat constituents of goat and sheep milk. Small Rumin Res 68, 42-63.

Sauvant D, P Bas. 2001. La digestion des lipides chez le ruminant. INRA Prod Anim 14, 303-310. 
Shingfield KJ, JM Griinari. 2007. Role of biohydrogenation intermediates in milk fat depression. Eur J Lipid Sci Technol 109, 799-816.

Silva MMC, MT Rodrigues, CAF Rodrigues, RH Branco, MI Leão, ACM Magalhães, RS Matos. 2007ª Efeito da suplementação de lipídios sobre a digestibilidade e os parâmetros da fermentação ruminal em cabras leiteiras. $R$ Bras Zootec 36, 246-256.

Silva MMC, MT Rodrigues, RH Branco, CAF Rodrigues, JLR Sarmento, AC Queiroz, SP Silva. 2007 . Suplementação de lipídios em dietas para cabras em lactação: consumo e eficiência de utilização de nutrientes. $R$ Bras Zootec 36, 257-267.

Teh TH, LT Trung, ZH Jia, TA Gipson, KB Ogden, TF Sweeney. 1994. Varying amounts of rumen-inert fat for high producing goats in early lactation. J Dairy Sci, 77, 253-258.

Udén P, PE Colucci, PJ Van Soest. 1980. Investigation of chromium, cerium and cobalt as markers in digesta rate of passage studies. $J$ Sci Food Agric 31, 625-632.
Ueda K, A Ferlay, J Chabrot, JJ Loor, Y Chilliard, M Doreau. 2003. Effect of linseed oil supplementation on ruminal digestion in dairy cows fed diets with different forage:concentrate ratios. J Dairy Sci 86, 3999-4007.

Van Soest PJ, JB Robertson, BA Lewis. 1991. Methods for dietary fiber and nonstarch polysacharides in relation to animal nutrition. J Dairy Sci 74, 3583-3597.

Yang SL, DP Bu, JQ Wang, ZY Hu, D Li, HY Wei, LY Zhou, JJ Loor. 2009. Soybean oil and linseed oil supplementation affect profiles of ruminal microorganisms in dairy cows. Animal 3, 1562-1569.

Zervas G, K Fegeros, K Koytsotolis, C Goulas, A Mantzios. 1998. Soy hulls as a replacement for maize in lactating dairy ewe diets with or without dietary fat supplements. Anim Feed Sci Technol 76, 65-75. 